\title{
Método dinamométrico para avaliação da escora em barcos tipo Catamaran
}

CDD. 20.ed. 796.023

797.14
Gustavo Ricardo SCHÜTZ*

Alessandro HAUPENTHAL* Marcel HUBERT*

Suzana Matheus PEREIRA* Helio ROESLER ${ }^{*}$
*Centro de Educação

Física, Fisioterapia e

Desportos, Universida-

de do Estado de Santa

Catarina

\section{Resumo}

Este estudo tem como objetivo propor e validar um método de medição da distância de escora ("De") e do momento de escora ("Me"), através do uso de plataformas de força para barcos tipo Catamaran. 0 novo método propõe a medição por dinamometria, com o posicionamento de plataformas de força abaixo de cada casco, e a mensuração das Forças de Reação do Solo (FRS). Com base nas equações clássicas da estática são determinadas as variáveis "De" e "Me". Para validar o método proposto, foi utilizada a validação concorrente, através da comparação com os valores obtidos por cinemetria através do sistema "Peak Motus". Para correlação entre os dois métodos optou-se pelo coeficiente de correlação intraclasse (ICC). Foram analisadas diferentes posturas em duas diferentes inclinações do barco, simulando o adernamento do barco. Os resultados encontrados apontaram alta correlação (ICC > 0,99) e pequeno erro médio (1,00\% para "De" e 0,96\% para "Me") entre os métodos. Com isto, o método proposto se mostrou válido e eficiente, pois mensurou as variáveis de maneira rápida e precisa e se torna importante à medida que facilita a análise e auxilia a velejadores e técnicos na tomada de decisões.

UnIteRMos: Biomecânica; Dinamometria; Vela; Escora.

\section{Introdução}

Quando se veleja no contravento principalmente em ventos de médios a fortes, surge um par de forças, uma força no velame e outra na bolina gerando um momento que faz o barco adernar (LEGG, MACKIE \& SMith, 1999; SCHмidT, 1990). Para evitar que o barco aderne excessivamente, o velejador cria um momento no sentido inverso ao citado utilizando para tal, seu peso a uma certa distância do centro de gravidade do barco. Esta ação do velejador é denominada "escora", a distância do centro de gravidade (CG) do velejador ao CG do barco é definida como distância de escora ("De"), e o momento gerado pelo velejador é definido como "momento de escora" ("Me"), sendo este o produto da "De" pela força peso do atleta (MACKIE, 2003; RoesLer, Haupenthal \& FaQUin, 2003; TAN Aziz, Spurway, Toh, Mackie, Wei, Wong, Fuss \& TeH, 2006). Segundo MaïsetTi, GuÉvel, IANCHKINE, LEGROS e BRISWALTER (2002), especialistas consideram que a capacidade de manter certa posição de escora pode ser fator determinante do desempenho, pois contribui para manter a velocidade do barco durante a prova e ainda, um grande fator a ser obtido em treinamentos é aumentar a tolerância à posição de escora (Ninimma, Wright, Shephard \& Clarke, 1977; Wright, Clarke, Niinimma \& Shephard, 1976). A escora ocorre, em barcos tipo Catamaran, com o auxílio de um cabo preso ao mastro e ligado próximo a cintura do velejador, definindo o trapézio.

Os barcos tipo Catamaran são caracterizados pela existência de dois cascos e dois velejadores, denominados proeiro e timoneiro, com diferentes funções, mas podendo os dois executar o trapézio. As classes "Hobie Cat 16" e a Olímpica Tornado são as mais importantes classes deste tipo de barco.

No Tornado (FIGURA 1), assim como em outros barcos, o cabo de trapézio é posicionado do mastro até um gancho fixo na "fralda" (FIGURA 1, no detalhe), na altura da cintura do atleta. $\mathrm{O}$ trapézio possibilita 
No detalhe a "fralda" ("trapeze harness") em que é ligado o cabo do trapézio.

Foto: dos Autores. ao velejador, quando necessário, manter todo o corpo fora do barco, com apoio dos pés na lateral do casco, o que possibilita um maior "Me". Deshors (1998) cita a necessidade de uma postura o mais alongada possível no prolongamento do barco, possibilitando progredir mais rapidamente.

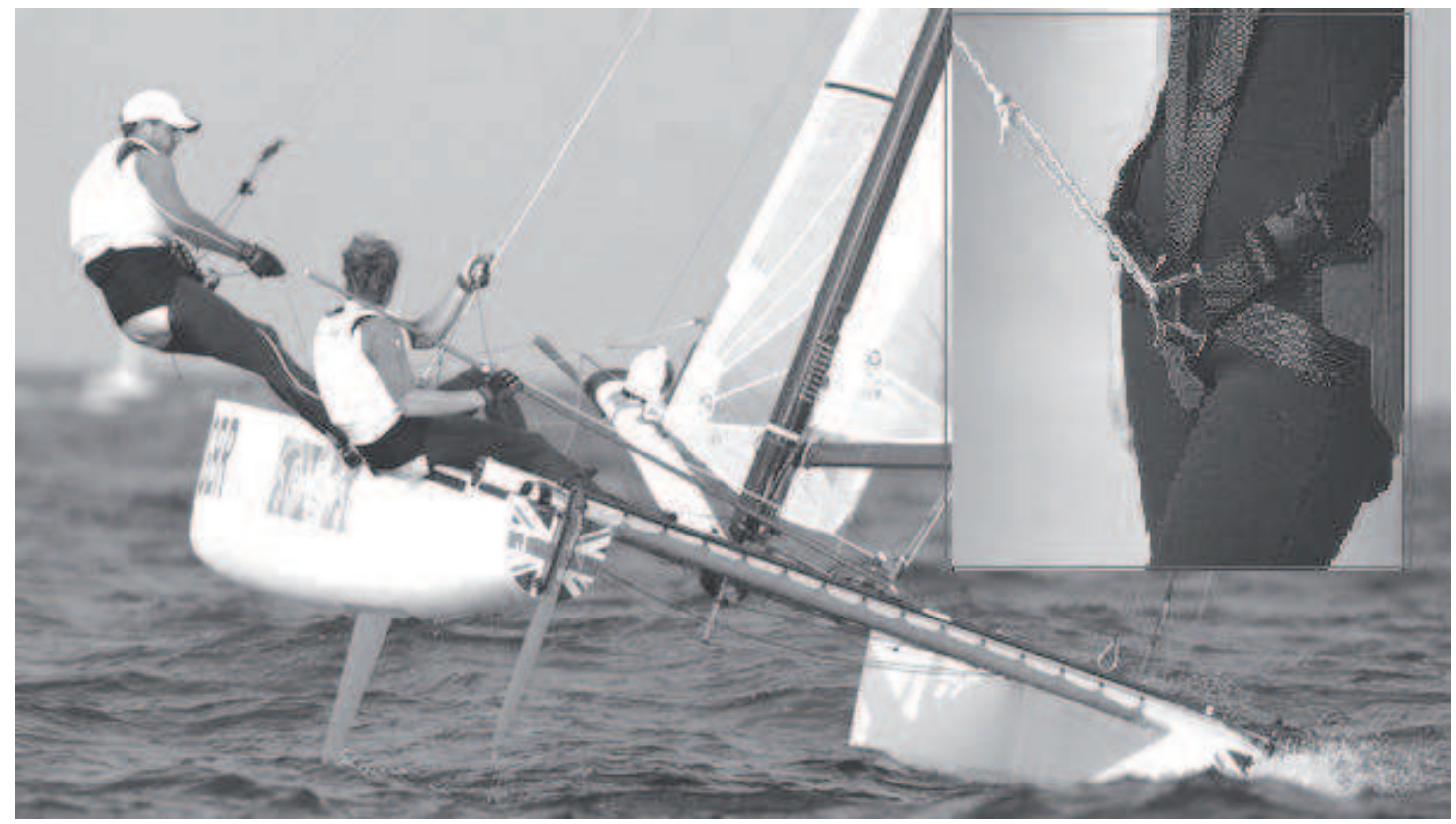

FIGURA 1 - Velejadores realizando a escora com auxílio do trapézio.

Estudos biomecânicos podem contribuir para responder tecnicamente dúvidas dos velejadores, referentes aos gestos motores durante o velejar, possibilitando aos técnicos e atletas escolher posturas confortáveis e eficientes para atingir maior rendimento. Métodos biomecânicos foram utilizados em estudos descrevendo e analisando as posiçôes utilizadas pelos velejadores em situaçôes laboratoriais, sendo que estas investigaçōes ocorreram principalmente em barcos do tipo monocasco, como os da classe Laser (BELLOT, RobHCongar, Briend, Mazer \& Lebars, 1979; BeIllot, Rochcongar, Gouard, Simonet, Briend \& Lebars, 1981; DeVito, Di Filippo \& MARChETTI, 1993; DuMORTIER \& IANChKINE, 2001; Le Deroff \& IANChKINE, 2001; Putnam, 1979). Poucos estudos (Marchetti, Figura \& Ricci, 1980; Roesler, Haupenthal \& Faquin, 2003) se preocuparam em analisar as posturas adotadas por velejadores em barcos Catamaran. MARChetTi, FigurA e Ricci (1980) citam que a exigência fisiológica das posturas com suporte do trapézio no "Hobie Cat" 16 como um exercício muito leve quando comparado à escora na classe Laser, podendo ser isto uma explicação a menor preocupação dos gestos nesta classe. Em contrapartida, RoesLer, HaUPENTHAL e FAQUiN (2003) citam a necessidade do conhecimento da eficiência mecânica e do conforto de cada postura para junto aos atletas determinar qual a melhor escolha para as situações de competição.
Ao realizar escora, independentemente da classe, $o$ velejador pode assumir diversas posturas, algumas mais confortáveis que outras, e cada uma dependendo da posição corporal e das características antropométricas do atleta gera uma "De" e um "Me" diferentes.

A mensuração da "De" e do "Me" pode ser feita por diferentes métodos. Um deles é através da cinemetria (BeILlot et al., 1979, 1981; Dumortier \& IANChKINE, 2001; Le Deroff \& IanchKIne, 2001; MaïsetTi, Guével, IANChKINe, Legros \& Briswalter, 2002; Putnam, 1979) onde o atleta é filmado (ou fotografado) em determinada posição, são calculadas as posiçōes dos centros articulares, com estas posições é calculado seu centro de gravidade (CG), a "De" e o "Me". Somente Marchetti, Figura e RICCI (1980) realizaram esta análise em um barco tipo Catamaran, mais precisamente, um "Hobie Cat" 16. Em alguns casos esta técnica pode ser realizada com uma única câmera se a filmagem ocorrer no plano perpendicular ao atleta e se não houver movimentos fora deste plano. Se estas condiçōes não forem satisfeitas, duas ou mais câmeras deverão ser utilizadas.

Outro métodoéo de dinamometria, com a mensuração das forças atuantes no movimento ou na posição estudada. Comumente mediçōes dinamométricas são realizadas em barcos tipo monocasco (exemplo: o barco da classe Laser), onde a cinta de escora é instrumentalizada com sensores os quais mensuram a força exercida pelos pés na cinta 
em diferentes posições adotadas. Os valores obtidos desta maneira são por vezes associados a variáveis fisiológicas, ao desempenho em regatas, a valores expressos pela máxima contração voluntária dos músculos extensores e flexores da perna, a prevenção de lesões e também a definiçōes de quais posturas e ajustes se tornam mais eficientes (AAGAARD, Beyer, Simonsen, Larsson, Magnussun \& KJaER, 1998; Blackburn, 1994; FeliCi, Rodio, MadafFari, ErColani \& Marchetti, 1999; LarsSon, Beyer, Bay, Blond, AagaArd \& KJAER, 1996; MACKIE \& LegG, 1999; MaCKIE, SANDERS \& LEGG, 1999; MAïsETTI et al., 2002; RIECKERT, 1993; TAN et al., 2006; VogiatZis, SpurWAY, JeNnETt, WiLSON

\section{Método}

\section{Dinamometria}

Os instrumentos utilizados foram plataformas de força desenvolvidas por RoEsLER (1997). O sistema é composto por duas plataformas extensométricas de $0,5 \times 0,5 \mathrm{~m}$. Sensibilidade de $2 \mathrm{~N}$, erro inferior a $1 \%$ e frequência natural de $60 \mathrm{~Hz}$. Como os esforços mecânicos gerados a partir do posicionamento do atleta no barco foram relativamente lentos, para o tratamento dos dados foi utilizado um filtro passa baixa tipo Butterworth de 0 a $25 \mathrm{~Hz}$. Foi utilizada a placa CIO-EXP-BR1IDGE de 16 canais como ponte de Weatstone e condicionamento de sinais, e conversor A/D CIO-DAS-16Jr com capacidade
\& SINCLAIR, 1996). Este valor de força é multiplicado pela "De" resultando no "Me", sendo que o CG do velejador é determinado por percentagem de sua altura e o CG do barco é referenciado no meio do barco.

Entretanto estes métodos não apresentam definições quanto sua fidedignidade, acurácia e erro na mensuração, além de não levar em consideração pontos similares a situação real. Tendo isto, este trabalho objetivou propor e validar um método de mensuração da distância e momento de escora por dinamometria, para barcos do tipo Catamaran, de forma fácil, precisa e fidedigna. para 16 canais e limite máximo de aquisição de $60 \mathrm{kHz}$, ambos da empresa Computer Boards. O processamento e leitura de valores obtidos se deram através do programa SAD32 (SiLVA \& ZARo, 2001). A frequência de aquisição adotada foi de 600 pontos por canal por segundo.

Durante a coleta de dados, as plataformas de força foram dispostas uma embaixo de cada casco, em duas situaçôes. Na primeira o velejador realizou as posturas de escora sem que houvesse inclinação do barco (plano: $0^{\circ}$ ), e em seguida um dos lados era elevado, simulando o adernamento do barco, gerando a situação inclinada (Inclinado: $5^{\circ}$ ). A representação das forças sobre o sistema é apresentada na FIGURA 2.

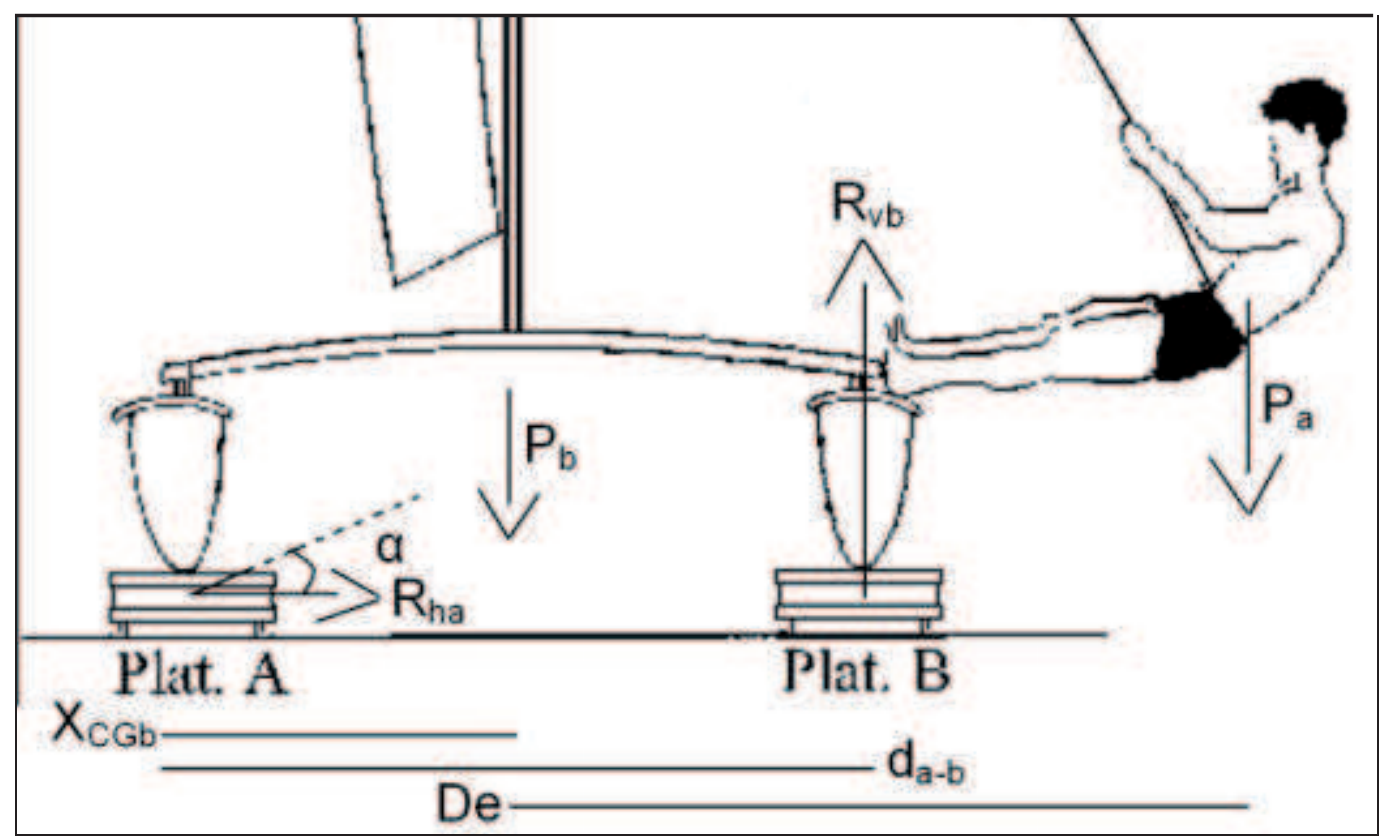

Onde:

$P=$ Peso do barco;

$\mathrm{X}_{\mathrm{CGb}}^{\mathrm{b}}=$ distância do CG do barco;

$\mathrm{R}_{\mathrm{b}}=$ componente vertical daFRS na plataforma B;

$\mathrm{d}_{\mathrm{a}}=$ distância entre as plataformas $\mathrm{A}$ e $\mathrm{B}$; $\cos \alpha=\operatorname{cosseno~do~ângulo~}$ formado com a elevação; $\mathrm{R}_{\mathrm{ha}}=$ componente horizontal da FRS na plataforma $A$; $\mathrm{h}_{\text {inc }}=$ altura da elevação; $\mathrm{P}_{\mathrm{a}}=$ peso do atleta.

FIGURA 2 - Representação das forças sobre o sistema (barco + velejador), visto de frente. 
Com os valores das forças de reação do solo (FRS) foram calculados o CG do barco (a); a "De" (b); e o
"Me" (c), com base nas equações clássicas da estática (BeEr \& Johnston Junior, 1977):
Onde:

$P_{b}=$ Peso do barco;

$X_{\mathrm{CGb}}=$ distância do CG do barco;

$\mathrm{R}_{\mathrm{b}}=$ componente vertical da FRS na plataforma B;

$d_{a-b}=$ distância entre as plataformas $A$ e $B$;

$\cos \alpha=$ cosseno do ângulo formado com a elevação;

$\mathrm{R}_{\mathrm{h}}=$ componente horizontal da FRS na plataforma A;

$h_{\text {inc }}=$ altura da elevação;

$P_{\text {inc }}=$ peso do atleta;

"De" = distancia de escora;

"Me" = Momento de escora.

(a)

$$
\begin{gathered}
\sum M a=0 \\
-\left(\mathrm{P}_{\mathrm{b}} \cdot \mathrm{X}_{\mathrm{CGb}}\right)+\left(\mathrm{R}_{\mathrm{vb}} \cdot \mathrm{d}_{\mathrm{a}-\mathrm{b}} \cdot \cos \alpha\right)=0 \\
\mathrm{X}_{\mathrm{CGb}}=\frac{\left(\mathrm{R}_{\mathrm{vb}} \cdot \mathrm{d}_{\mathrm{a}-\mathrm{b}} \cdot \cos \alpha\right)}{\mathrm{P}_{\mathrm{b}}}
\end{gathered}
$$

$$
\sum \mathrm{Ma}=0
$$

$$
\begin{array}{r}
-\left(P_{b} \cdot X_{C G b}\right)+\left(R_{v b} \cdot d_{a-b} \cdot \cos \alpha\right)-\left(R_{h a} \cdot h_{i n c}\right)-\left(P_{a} \cdot D e\right)=0 \\
D e=\frac{\left(P_{b} \cdot X_{C G b}\right)-\left(R_{v b} \cdot d_{a-b} \cdot \cos \alpha\right)+\left(R_{h a} \cdot h_{i n c}\right)}{P_{a}}
\end{array}
$$

(c)

\section{Cinemetria}

Para a cinemetria utilizou-se uma filmadora SVHS, com frequência de aquisição de $60 \mathrm{~Hz}$, ocorrendo a aquisição de forma simultânea a dinamometria. A análise foi realizada no "software" do sistema "Peak Motus" (Peak Performance Technology, 1998). Para a determinação do centro de gravidade do atleta foi utilizado o modelo proposto por Clauser, MCCONVILLE e YounG (1969) para a definição das massas e das propriedades geométricas e inerciais de cada segmento.

Utilizou-se um modelo anatômico com a determinação dos seguintes pontos para o atleta: articulação-têmporomandibular, acrômio, epicôndilo lateral do úmero, processo estilóide lateral da ulna, crista ilíaca, epicôndilo lateral do fêmur, maléolo lateral da fíbula, $\mathrm{V}$ metatarso distal.

Nas situações propostas foi feita a análise bidimensional, com os devidos calibradores para cada eixo de coordenadas. A posição da câmera de vídeo foi perpendicular ao plano longitudinal do barco à frente do seu CG, anteriormente calculado por dinamometria, tanto para o barco plano quanto na situação inclinada. Foi utilizado como referência o CG do barco ao invés de utilizar como referência o meio do barco, como utilizado em outros trabalhos (BEILLT et al., 1979, 1981; De Vito, Di Filippo \& MarChetTI, 1993; DUMORTIER \& IANCHKINE, 2001; Le DerofF \& IANCHKINE, 2001; MACKIE, 2003; MAÏSETTI et al., 2002; MARCHETTI, Figura \& Ricci, 1980; PUtNAM, 1979; TAN et al., 2006).

\section{Sujeitos}

Participou desta pesquisa um sujeito do gênero masculino, 30 anos, 76,0 kg de massa, 1,86 m de estatura, velejador da classe e participante de regatas internacionais.
$\mathrm{Me}=\mathrm{De} \cdot \mathrm{P}_{\mathrm{a}}$

\section{Desenvolvimento/Procedimentos}

As plataformas foram dispostas embaixo de um barco da classe "Hobie Cat" 16. Nesta etapa pode-se mensurar o peso do barco e a localização do CG.

Foram então simuladas diversas posturas em dois diferentes comprimentos de trapézio (Alto e Baixo) e duas inclinações do barco (plano e inclinado), totalizando 36 posições. As posturas diferenciavam quanto à flexão/extensão dos joelhos (1200 a 1800), quadril em flexão/extensão (60 a 1550$)$ e posicionamento dos braços (juntos ao corpo a estendidos acima da cabeça). Em trapézio Alto o cabo de trapézio permanece no comprimento mínimo $(5,83 \pm$ $0,05 \mathrm{~m}$ ) e em trapézio Baixo o cabo permanece no comprimento máximo $(6,03 \pm 0,05 \mathrm{~m})$.

O estudo foi aprovado pelo Comitê de Ética em Pesquisa da Universidade do Estado de Santa Catarina, sob o número de referência 46/2007.

\section{Tratamento estatístico}

Os dados foram analisados através do pacote estatístico SPSS versão 13.0. Estatística descritiva (média e desvio padrão) e inferencial (índice de correlação intraclasse - ICC, do inglês "Intraclass correlation coeficient"; dados normais pelo teste de normalidade de Shapiro-Wilk) foram utilizadas. O nível de significância foi de 5\% (p < 0,05). Para interpretação dos valores do ICC, foi utilizada a escala de valores sugerida por SzKLO e Nieto (2000), onde valores acima de 0,75 indicam repetibilidade excelente; valores entre $0,40-0,75$, repetibilidade satisfatória e valores abaixo de 0,40 repetibilidade pobre. 
A mensuração por cinemetria foi considerada como o "real" valor, sendo o erro entre os métodos expressos em percentagem (valor mensurado pordinamometria-valor "real" por cinemetria / 100), determinando o erro médio e máximo.

\section{Resultados}

A massa e o CG do barco para as duas situaçôes são apresentados na TABELA 1.

Determinado o CG do barco, foi determinado o CG do velejador, por dinamometria e cinemetria, e então calculados "De" e "Me". Os resultados são apresentados na TABELA 2.

Considerando a cinemetria com o valor "real", foi determinada a correlação entre os métodos pelo coeficiente de correlação intraclasse (ICC) apresentado na TABELA 3. Juntamente são apresentados o erro médio e o erro máximo verificados.

Os valores do ICC representam correlação muito alta e repetibilidade excelente entre os métodos utilizados. O erro médio foi de aproximadamente $1 \%$, sendo que este erro possa ser resultado do erro existente do sistema de dinamometria (<1\%) (RoESLER, 1997) e/ ou da cinemetria $(<1 \%$ ) (Peak Performance TechNOLOGY, 1998), que é próximo do valor encontrado.

TABELA 1 - Valores para determinação do centro de gravidade do barco.

\begin{tabular}{lcc}
\hline Massa do barco $(\mathrm{kg})$ & \multicolumn{2}{c}{145,0} \\
Distância entre os vértices do barco $(\mathrm{m})$ & \multicolumn{2}{c}{2,17} \\
\cline { 2 - 3 } & Plano & Inclinado \\
Elevação de um dos lados $(\mathrm{m})$ & 0,00 & 0,20 \\
Ângulo de inclinação $\left(^{\circ}\right)$ & 0 & 5 \\
Centro de Gravidade do barco $(\mathrm{m})$ & 1,07 & 0,98 \\
\hline
\end{tabular}

TABELA 2 - Valores da distância de escora e momento de escora para as posturas $(n=36)$.

\begin{tabular}{|c|c|c|c|c|}
\hline & \multicolumn{2}{|c|}{ “De" (Distância de escora) (m) } & \multicolumn{2}{|c|}{ "Me" (Momento de escora) (N.m) } \\
\hline & Cinemetria & Dinamometria & Cinemetria & Dinamometria \\
\hline \multicolumn{5}{|l|}{ Barco plano } \\
\hline Trapézio alto $(\mathrm{n}=9)$ & $\mathbf{2 , 1 7} \pm 0,27$ & $\mathbf{2 , 1 5} \pm 0,27$ & $1615 \pm 202$ & $1599 \pm 201$ \\
\hline Trapézio baixo $(\mathrm{n}=9)$ & $2,27 \pm 0,26$ & $2,24 \pm 0,25$ & $1693 \pm 192$ & $1699 \pm 188$ \\
\hline \multicolumn{5}{|l|}{ Barco inclinado } \\
\hline Trapézio alto $(\mathrm{n}=9)$ & $\mathbf{2 , 2 8} \pm 0,23$ & $\mathbf{2 , 3 0} \pm 0,24$ & $1702 \pm 172$ & $1713 \pm 175$ \\
\hline Trapézio baixo $(\mathrm{n}=9)$ & $2,36 \pm 0,16$ & $\mathbf{2 , 3 7} \pm 0,19$ & $1762 \pm 120$ & $\mathbf{1 7 6 8} \pm 138$ \\
\hline
\end{tabular}

TABELA 3 - Correlação e erro entre os métodos.

\begin{tabular}{lccc}
\hline & ICC & Erro médio & Erro máximo \\
\hline "De" Distância de escora & $\mathbf{0 , 9 9}$ & $1,00 \%$ & $1,65 \%$ \\
"Me" Momento de escora & $\mathbf{0 , 9 9}$ & $0,96 \%$ & $1,55 \%$ \\
\hline
\end{tabular}




\section{Discussão}

Um corpo rígido livre aprecia um máximo seis graus de liberdade: três rotações e três translações. Matematicamente, todo o movimento que o corpo executar pode ser descrito como uma combinação destes. Enquanto a sustentação e a estabilidade na terra podem ser obtidas à custa de um ou mais grau de liberdade, um barco mostra componentes de todos os seis (BOJSEN-MøLLER \& BOJSEN-MølLER, 1999). Uma terminologia náutica comum tem nomes próprios para todos os seis, sendo os movimentos de rotação chamados adernamento (do inglês, "rolling or heel"), arfagem ("pitching") e guinada ("yawing"); e as translaçōes de submersão ("heaving"), deslocamento ("swaying") e aceleração ("surging"), definidos Heimberg (1994) e SjøgaARD (1999), adaptados para o português por SCHÜTZ (2006).

Em barcos nos quais o peso da tripulação, relativo ao peso do barco, é grande, a posição adotada pela tripulação é importante para o contrapeso e alinhamento longitudinal do barco e desse modo, fazer com o barco plane. Para contrapor o adernamento do barco, para maximizar o contrapeso e o alinhamento horizontal, a tripulação adota uma posição denominada de escora, alvo de diversos estudos (AAGAARD et al., 1998; BeILLOT et al., 1979, 1981; BLACKBURN, 1994; De Vito, Di Filippo \& Marchetti, 1993; DuMORTIER \& IANCHKINE, 2001; FeliCi et al., 1999; Larsson et al., 1996; Le Deroff \& IANChKine, 2001; Mackie, 2003; Mackie \& LegG, 1999; Mackie, Sanders \& LegG, 1999; Menezes, Schütz, Cerutti, Carneiro, Fontana \& Roesler, 2007; Putnam, 1979; RieCKerT, 1993; RoEsler, Haupenthal \& FAQUIN, 2003; TAN et al., 2006; VogiaTZIS et al., 1996).

A tripulação constitui um tipo de balastro que desloca o CG do sistema total (barco e tripulação) para preservar o alinhamento longitudinal. A força do vento que age no centro da vela (centro vélico) e a força de arrasto resistindo da superfície da água e na bolina (centro de carena) criam um momento que deve ser balanceado pelo par de vetores do centro de flutuabilidade e do CG (BOJSEN-MølLER \& BOJSEN-MøLleR, 1999).

Movendo-se longitudinalmente a tripulação pode mudar a distância entre os centros e assim ajustar o momento de contrapeso perfeito ("Me") evitando o adernamento excessivo do barco. Com o adernamento em excesso o barco desenvolve um componente dirigido descendente que faz a passagem através da água cada vez mais pesada, difícil. Para planar o barco deve ser mantido alinhado longitudinalmente, não adernando mais que de $0-10^{\circ}$ (BLACKBURN, 1994) ou

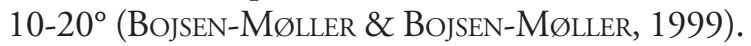

A posição do CG em relação ao centro de carena é fator determinante da estabilidade e flutuabilidade da embarcação. $O$ centro de gravidade situa-se próximo à mesma vertical que o centro de carena, movendo-se ao longo desta linha, segundo a variação do peso da embarcação e de sua carga, e segundo a distribuição desse peso (CiviTA, 1975), sendo neste caso, a ação de escora. Foi observado que quando adernamos (inclinamos) o barco, ocorre a variação do CG do barco (TABELA 1), e esta variação deve ser considerada para a exata determinação de "De" e "Me". BojSen-Møleer e BojSen-Møller (1999) relatam a dependência do grau de adernamento para a obtenção do "Me". Exposta a importância e função da localização do CG do barco, ao contrário do uso do meio do barco como referência, como verificados em estudos sobre a escora (BEILLOT et al., 1979, 1981; DE Vito, Di Filippo \& Marchetti, 1993; Dumortier \& IanchKine, 2001; Le Deroff \& IanchKine, 2001; Mackie, 2003; Maïsetti et al., 2002; MARChetTI, Figura \& Ricci, 1980; Putnam, 1979; Tan et al., 2006), o novo método apresentado com o uso de plataformas de força possibilita a localização do CG do barco, tornando mais preciso a determinação da "De" e, consequentemente, do "Me" produzido pelo velejador.

Entre os métodos, o novo método proposto proporciona uma coleta relativamente rápida e fácil, podendo gerar respostas rápidas das variáveis devido ao menor processo de análise que a cinemetria. No novo método é possível analisar posturas com variaçóes além de somente um plano dimensional, sendo que no mesmo processo por cinemetria seria necessário o uso de uma análise tridimensional, com duas ou mais câmeras, maior tempo e também maior custo. Determinada a eficiência do novo método, este se apresenta de grande valor e aplicabilidade quando comparado à cinemetria, levando em conta os processos metodológicos de instrumentação, procedimentos, coleta e análise de dados.

A análise da variação angular não é possível com o novo método, mas esta é considerada fator de menor importância para o desempenho do velejador (MACKIE, 2003). "De" e "Me" são considerados melhores indicativos e apresentam correlação com melhores resultados (MACKIE, 2003; MAḮSETTI et al., 2002; Roesler, Haupenthal \& Faquin, 2003; Tan et al., 2006). Achando-se necessária a análise da variação angular, uma alternativa válida e mais prática que a cinemetria seria a utilização de eletrogoniômetros, aparelhos que possibilitam a mensuração da variação 
angular de articulações em um plano (NIGG \& HerzoG, 1999), sendo relevante a análise da variação angular de joelho e quadril realizada apenas no plano sagital (Beillot et al., 1979; Dumortier \& IanChKine, 2001; MAïsETTI et al., 2002).

Convém notar que, mesmo com as vantagens apresentadas no novo método, ainda não representa a situação real da Vela, onde não são reproduzidas as condiçôes do vento e da água, pontos que influem no desempenho do barco e velejador (SHEPHARD, 1997). No entanto, quando não seja possível a avaliação na água, é recomendada a utilização de bancos de escora ("hiking bench"), um ergômetro, o qual permite a reprodução das posturas de escora, como demonstrado por Tillman (2005), ou de forma mais especifica com o uso do próprio barco, como desenvolvido no método proposto. As condições de água e vento são possíveis de serem simuladas no futuro com base no novo método proposto.

Ë possível utilizar o novo método no desenvolvimento de diversas pesquisas, associadas ou não a variáveis de outra natureza, como fisiológicas, por exemplo.

\title{
Conclusão
}

O método desenvolvido mostrou se eficiente para o fim proposto. Nas diversas posições do velejador e do barco, o centro de gravidade do velejador, a distância de escora e o momento de escora foram mensurados de forma relativamente fácil, precisa e eficiente. Os valores encontrados por dinamometria apresentaram alta correlação com os obtidos por cinemetria e baixo erro, o que afere precisão ao novo método. E por fim, a possibilidade de definição do centro de gravidade do barco garante maior confiabilidade para a determinação das demais variáveis.

\begin{abstract}
Dynamometric method for the evaluation of hiking in Catamaran boats

This study aimed to propose and to validate a method for the measurement of hiking distance $(\mathrm{Hd})$ and hiking moment $(\mathrm{Hm})$ in Catamaran boats, through the use of force plates. This new method proposes the measurement by dynamometry, with the positioning of a force plate below each boat hoof to measure the Ground Reaction Forces (GRF). Based on the classic static equations Hd and Hm were determined. In order to validate the method a validation of competing was carried out through the comparison with kinematic values measured through the Peak Motus system. Intraclass correlation coeficient was used. Different positions in two different inclinations of the boat, simulating the boat heeling, had been analyzed. The results showed a high correlation (ICC > 0.99) and a small average error $(1.00 \%$ for $\mathrm{Hd}$ and $0.96 \%$ for $\mathrm{Hm}$ ) between the methods. Thus, the new method seems to be valid and efficient since it measured the variables in a fast and precise way, facilitating the analysis and assisting sailors and coaches on decision taking.
\end{abstract}

UnITERMS: Biomechanics; Dynamometry; Sailing; Hiking.

\section{Referências}

AAGAARD, P.; BEYER, N.; SIMONSEN, E.B.; LARSSON, B.; MAGNUSSUN, S.P.; KJAER, M. Isokinetic muscle strength and hiking performance in elite sailors. Scandinavian Journal of Medicine \& Science in Sports, Copenhagen, v.8, n.3, p.138-44, 1998.

BEER, F.P.; JOHNSTON JUNIOR, E.R. Vector mechanics for engineers: statics. New York: McGraw-Hill, 1977. 
BEILLOT, J.; ROBHCONGAR, P.; BRIEND, G.; MAZER, J.; LEBARS, R. Approche biomecanique de la position de rappel. Lyon Mediterranée Medical, Paris, v.5, p.1279-83, 1979.

BEILLOT, J.; ROCHCONGAR, P.; GOUARD, P.; SIMONET, J.; BRIEND, G.; LE BARS, R. Le rappel sur Finn: Approche biomecanique. Cinésiologie, Paris, v.80, p.179-91, 1981.

BLACKBURN, M. Physiological responses to 90 min of simulated dinghy sailing. Journal of Sports Sciences, London,v.12, n.4, p.383-90, 1994.

BOJSEN-MØLLER, F; BOJSEN-MØLLER, J. Biomechanics of sailing. In: SJØGAARD, G. Sailing \& science: in an interdisciplinary perspective. Copenhagen: Institute of Exercise and Sports Science/University of Copenhagen, 1999. CIVITA, V. Dicionário ilustrado de navegação a vela. São Paulo: Abril Cultural, 1975.

CLAUSER, C.E.; McCONVILLE, J.T.; YOUNG, J.W. Weight, volume, centre of mass segments of the human body. AMRL Technical Report (TR 69 - 70). Local: Wright-Patteron Air Force Base, 1969.

DE VITO, G.L.; DI FILIPPO, F; MARCHETTI, M. Hiking mechanics in Laser athletes. Medical Science Research, Barking, v.10, n.23, p.859-61, 1993.

DESHORS, M. O grande livro da vela: aventura e prática da navegação. Lisboa: Chaves Ferreira, 1998.

DUMORTIER, B.; IANCHKINE, P. Mesure du couple de rappel en 470. 2001. Disponível em: <http://www.env. jeunesse-sports.fr>. Acesso em: 26 jul. 2004.

FELICI, F.; RODIO, A.; MADAFFARI, A.; ERCOLANI, L.; MARCHETTI, M. The cardiovascular work of competitive dinghy sailing. The Journal of Sports Medicine and Physical Fitness, Torino, v.39, n.4, p.309-14, 1999.

HEIMBERG, F. Untersuchungen zum operationellen Einsatz des Differential GPS. Doktor dissertation, Universität Hannover, 1994. LARSSON, B.; BEYER, P.; BAY, P.; BLOND, L.; AAGAARD, P.; KJAER, M. Exercise performance in elite male and female sailors. International Journal of Sports Medicine, Stuttgart, v.17, p.504-8, 1996.

LE DEROFF, J.Y.; IANCHKINE, P. Mesure du couple de rappel en laser. 2001. Disponível em: <http://www.env. jeunesse-sports.fr>. Acesso em: 26 jul. 2004.

LEGG, S.; MACKIE, H.; SMITH, P. Temporal patterns of physical activity in Olympic dinghy racing. The Journal of Sports Medicine and Physical Fitness, Torino, v.39, n.4, p.315-20, 1999.

MACKIE, H. Useful biomechanics for sailing: development of technique analysis protocol for Europe and laser sailors. In: HUMAN PERFORMANCE IN SAILING CONFERENCE, 2003, Auckland. Proceedings... Auckland: [s.ed.],2003. p. 71-5.

MACKIE, H.; LEGG, S. Preliminary assessment of force demands in laser racing. The Australian Journal of Science and Medicine in Sports, Pennant Hills, v.2, n.1, p.78-85, 1999.

MACKIE, H.; SANDERS, R.; LEGG, S. The physical demands of Olympic yacht racing. The Australian Journal of Science and Medicine in Sports, Pennant Hills, v.2, n.4, p.375-88, 1999.

MAÏSETTI, O.; GUÉVEL, A.; IANCHKINE, P.; LEGROS, P.; BRISWALTER, J. Le maintien de la position de rappel en dériveur solitaire. Aspects théoriques et propositions méthodologiques d'évaluation de la fatigue musculaire. Science \& Sports, Paris, v.17, p.234-46, 2002.

MARCHETTI, M.; FIGURA, F; RICCI, B. Biomechanics of two Fundamentals sailing potures. Journal of Sports Medicine, Baltimore, v.20, p.325-32, 1980.

MENEZES, F.S.; SCHÜTZ, G.R.; CERUTTI, P.R.; CARNEIRO, L.C.; FONTANA, H.B.; ROESLER, H. Analysis of spine movements in hiking on sailing: a preliminary study In: INTERNATIONAL SYMPOSIUM ON BIOMECHANICS IN SPORTS, 25., 2007, Ouro Preto. Proceedings... Belo Horizonte: Universidade Federal de Minas Gerais, 2007. p.125-28. NIGG, B.M.; HERZOG, W. Biomechanics of the muscle-skeletal system. 2nd. ed. Local, John Wiley, 1999.

NIINIMMA, V.; WRIGHT, G.; SHEPHARD, R.J.; CLARKE, J. Characteristics of the successful dinghy sailor. Journal of Sports Medicine, Baltimore, v.17, p.83-96, 1977.

PEAK PERFORMANCE TECHNOLOGY. Peak start manual. Local: editora, 998.

PUTNAM, C.A. A mathematical model of hiking positions in a sailing dinghy. Medicine and Science in Sport, Madison, v.11, n.3, p.288-92, 1979 .

RIECKERT, H. Sports medical aspects of sailing and windsurf. Deutsche Zeitschrift für Sportmedizin, Köhn, v.44, p.301-3, 1993. ROESLER, H. Desenvolvimento de plataforma subaquática para medições de forças e momentos nos três eixos coordenados para utilização em biomecânica. 1997. 193f. Tese (Doutorado em Engenharia Mecânica) - Universidade do Estado do Rio Grande do Sul, Porto Alegre, 1997.

ROESLER, H.; HAUPENTHAL, A.; FAQUIN, A. Desenvolvimento de técnica dinamométrica para mensuração do momento de escora em veleiros do tipo Catamaran. In: CONGRESSO BRASILEIRO DE BIOMECÂNICA, 10., Ouro Preto. Anais... Ouro Preto: SBB, 2003. v1, p.399-402. 
SCHMIDT, J.G. Do optimist ao iate de oceano: tudo sobre vela. Rio de Janeiro: Ediçôes Marítimas, 1990.

SCHÜTZ, G.R. Desenvolvimento e validação de sistema de aquisição de imagens na vela. 2006. Dissertação (Mestrado em Ciências do Movimento Humano) - Universidade do Estado de Santa Catarina, Florianópolis, 2006.

SHEPHARD, R.J. Biology and medicine of sailing: an update. Sports Medicine, Auckland, v.23, n.6, p.350-6, 1997.

SILVA L.; ZARO, M.A. SAD versão 3.2: sistema de aquisição de dados, manual de operação. Porto Alegre: UFRGS, 2001. (Caderno Técnico de Engenharia Mecânica CT07 - DEMEC).

SJØGAARD, G. Sailing \& science: in an interdisciplinary perspective. Copenhagen: Institute of Exercise and Sports Science, 1999.

SZKLO, M.; NIETO, F. J. Epidemiology, beyond the basics. Gaithersburg: Aspen, 2000.

TAN, B.; AZIZ, A.R.; SPURWAY, N.C.; TOH, C.; MACKIE, H,; WEI, X.; WONG, J.; FUSS, F.K.; TEH, K.C. Indictors of maximal hiking performance in laser sailors. European Journal of Applied Physiology, Berlin, v.98, p.160-76, 2006. TILLMAN, D. The complete book of laser sailing. New York: McGraw-Hill, 2005.

VOGIATZIS, I.; SPURWAY, N.C; JENNETT, S.; WILSON, J.; SINCLAIR, J. Changes in ventilation related to changes in electromyography activity during repetitive bouts exercise in simulated dinghy. European Journal of Applied Physiology, Berlin, v.72, p.195-203, 1996.

WRIGHT, G.; CLARKE, J.; NIINIMAA, V.; SHEPHARD, R.J. Some reactions to a dry-land training programme for dinghy sailors. British Journal of Sports Medicine, Loughbough, v.10, n.1, p.4-10, 1976.

ENDERECCO

Gustavo Ricardo Schütz Laboratório de Pesquisas em Biomecânica Aquática CEFID/UDESC

R. Pascoal Simone, 358 - Coqueiros 88080-350 - Florianópolis - SC - BRASIL e-mail: gugaschutz@hotmail.com
Recebido para publicação: 10/12/2008

Revisado em: 14/09/2009

Aceito: 14/09/2009 